\title{
Accurate Approximations for the Capacity Distribution of OFDM-Based Spatial Multiplexing
}

\author{
Matthew R. McKay ${ }^{\dagger}$, Peter J. Smith ${ }^{\ddagger}$, Himal A. Suraweera**, Iain B. Collings* \\ ${ }^{\dagger}$ Department of Electronic and Computer Engineering, Hong Kong University of Science and Technology, Hong Kong \\ ${ }^{\ddagger}$ Department of Electrical and Computer Engineering, University of Canterbury, Christchurch, New Zealand \\ ** Department of Electrical and Computer Systems Engineering, Monash University, Melbourne, Australia \\ ${ }^{*}$ Wireless Technologies Laboratory, ICT Centre, CSIRO, Sydney, Australia
}

\begin{abstract}
We derive new analytic approximations to the capacity distribution of frequency-selective Rayleigh fading MIMO channels. The results apply specifically to OFDM-based spatial multiplexing. In particular, we present a new closed-form Gaussian approximation which yields very high accuracy in many scenarios. For the low SNR regime, we also present a new simpler closed-form Gamma approximation, which we show to be even more accurate than Gaussian in this case. Our approximations are based on new exact closed-form expressions which we derive for the variance of the mutual information which, in contrast to previous results, apply for systems with both arbitrary numbers of antennas and arbitrary-length channel delay profiles.
\end{abstract}

\section{INTRODUCTION}

Multiple-input multiple-output (MIMO) antenna technology has emerged as an effective technique for significantly improving the capacity of wireless communication systems. A great deal of work has been done on analyzing the MIMO capacity in various flat-fading channel scenarios, since the pioneering work of [1] and [2]. In particular, the mean (ergodic) capacity has now been comprehensively investigated (e.g. see [3-9]). In addition, the outage capacity has also been investigated for flat-fading channels. This is a relevant capacity measure for systems with stringent delay constraints and also provides information about the system diversity [10]. With the exception of the exact two/three antenna results in $[11,12]$, outage capacity analysis has typically involved approximating the distribution of the mutual information, since exact closed-form solutions are not forthcoming. It has been shown that the Gaussian distribution gives a good approximation in many cases $[3,6]$.

In this paper, we consider frequency-selective MIMO channels, which are applicable for many current high data-rate wireless systems. For these channels, there are relatively few analytic MIMO capacity results. The ergodic capacity was considered in $[10,13,14]$ and $[15,16]$, assuming Rayleigh and Rician channels respectively. The most common approach is to derive the capacity in the context of orthogonal frequencydivision multiplexing (OFDM) based spatial multiplexing systems. It has been shown that the ergodic capacity of these systems can be easily obtained by summing the flat-fading ergodic MIMO capacity of each OFDM subcarrier. In contrast, the outage capacity does not decompose in this way.

Calculating the outage capacity for frequency-selective channels is difficult due to the non-negligible correlations between subcarrier channel matrices. As such, the investigation of outage capacity has usually been performed using simulation studies $[10,17]$. It appears that the only current analytical outage capacity results for frequency-selective MIMO channels are presented in [18] and [19]; both of which derived a Gaussian approximation to the mutual information using asymptotic methods. Specifically, in [18], the mean and variance of the mutual information were derived for asymptotically large channel lengths. In [19], the mean and variance were derived for finite-length channels, but assuming infinite numbers of transmit and receive antennas.

In this paper, we consider OFDM-based spatial multiplexing systems with finite numbers of antennas, and operating over channels with finite delay spreads. We derive new exact closed-form expressions for the mutual information variance, and give an explicit reduced formula for the specific case of single-input single-output (SISO) systems. Simplified closedform expressions are derived for the variance in the low signal-to-noise ratio (SNR) regime.

Based on the new analytic variance results (along with known analytic mean results), we then present new approximations to the mutual information distribution of OFDMbased spatial multiplexing systems. In particular, we present a new closed-form Gaussian approximation, which is shown to be extremely accurate for many different system and channel scenarios. In the low SNR regime, we also present a new analytic Gamma approximation, which we show to be more accurate than the Gaussian approximation in this case.

\section{OFDM-Based Spatial Multiplexing Systems}

\section{A. Channel and Signal Model}

We consider a single-user OFDM-based spatial multiplexing system employing $N_{t}$ transmit antennas, $N_{r}$ receive antennas, and $N$ subcarriers. The channel is assumed to be frequency-selective and is modeled as a length- $L$ finite impulse-response (FIR) filter (as in [10,13]), for which the discrete-time input-output relation is given by [13]

$$
\mathbf{y}[q]=\sum_{p=0}^{L-1} \sigma_{p}^{2} \mathbf{H}[p] \mathbf{x}[q-p]+\mathbf{n}[q]
$$

where $\mathbf{x}[q] \in \mathcal{C}^{N_{t} \times 1}$ is the signal vector transmitted at sample index $q, \mathbf{y}[q] \in \mathcal{C}^{N_{r} \times 1}$ is the corresponding received signal vector, and $\mathbf{n}[q] \in \mathcal{C}^{N_{r} \times 1}$ is the noise vector containing independent elements $\sim \mathcal{C N}(0,1)$. Also, $\sigma_{p}^{2}$, for $p=0, \ldots, L-1$, represents the channel power delay profile, and is normalized according to $\sum_{p=0}^{L-1} \sigma_{p}^{2}=1$.

The $N_{r} \times N_{t}$ matrices $\mathbf{H}[p]$, for $p=0, \ldots, L-1$, represent the MIMO channel impulse response. These matrices 


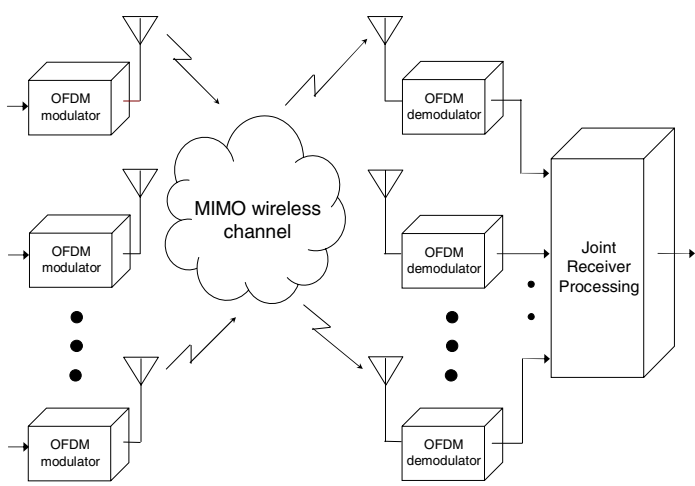

Fig. 1. Block diagram of an OFDM-based spatial multiplexing system.

are assumed to be mutually uncorrelated. The channel is assumed quasi-static, remaining constant for the duration of a codeword, but changing independently from codeword to codeword. The channel elements are assumed to be i.i.d. Rayleigh fading, in which case each $\mathbf{H}[p]$ contains independent elements $\sim \mathcal{C N}(0,1)$. The channel matrices are assumed to be known perfectly at the receiver, but are unknown at the transmitter.

At the transmitter, the time-domain input sequence $\mathbf{x}[q]$ is generated as $N_{t}$ parallel OFDM symbols, as shown in Fig. 1. The symbols for each antenna are OFDM modulated using an $N$-point inverse fast-Fourier transform (IFFT) prior to transmission. At the receiver, OFDM demodulation is performed at each receive antenna using an $N$-point FFT. A key advantage of OFDM-based spatial multiplexing is that equalization is simple, since the frequency-selective MIMO channel is transformed into $N$ orthogonal flat-fading MIMO subchannels via the IFFT/FFT processing.

To maintain orthogonality in the presence of intersymbol interference caused by multipath, OFDM systems typically employ a cyclic prefix extension. Assuming that the cyclic prefix is longer than the delay spread of the channel, we can write the equivalent frequency domain input-output model for OFDM-based spatial multiplexing as follows

$$
\mathbf{r}_{k}=\mathbf{H}_{k} \mathbf{a}_{k}+\mathbf{n}_{k}, \quad k=0, \ldots, N-1
$$

where $\mathbf{a}_{k}$ is the transmitted vector for the $k$ th subcarrier, satisfying $E\left[\mathbf{a}_{k} \mathbf{a}_{k}^{\dagger}\right]=\frac{\gamma}{N_{t}} \mathbf{I}_{N_{t}}, \mathbf{r}_{k}$ is the received vector for the $k$ th subcarrier, and $\mathbf{n}_{k}$ is the corresponding complex AWGN vector satisfying $E\left[\mathbf{n}_{k} \mathbf{n}_{\ell}^{\dagger}\right]=\mathbf{I}_{N_{r}} \delta[k-\ell]$, where $\delta[\cdot]$ is the kronecker-delta function. Also, $\mathbf{H}_{k}$ is the $k$ th subcarrier channel matrix given by

$$
\mathbf{H}_{k}=\sum_{p=0}^{L-1} \sigma_{p}^{2} \mathbf{H}[p] \exp \left(-j 2 \pi \frac{k}{N} p\right)
$$

containing independent entries $\left(\mathbf{H}_{k}\right)_{i, j} \sim \mathcal{C N}(0,1)$. Note that due to the finite-length impulse response, correlation exists between different subcarrier channel matrices. Using (3), the correlation coefficients between the channel elements on two arbitrary subcarriers $k$ and $\ell$ is easily derived as follows (see also [20])

$$
\rho_{k-\ell}=E\left[\left(\mathbf{H}_{k}\right)_{i, j}\left(\mathbf{H}_{\ell}\right)_{i^{\prime}, j^{\prime}}^{*}\right]
$$

$$
=\sum_{p=0}^{L-1} \sigma_{p}^{2} e^{-j 2 \pi(k-\ell) p / N} \delta\left[i-i^{\prime}\right] \delta\left[j-j^{\prime}\right]
$$

for all $i, j, i^{\prime}, j^{\prime}$. As expected, these frequency correlation coefficients depend only on the difference between subcarriers (i.e. $k-\ell$ ), and not on the subcarriers themselves.

Note that with the above model, the SNR per receive antenna per subcarrier (henceforth referred to as 'the SNR') is given by $\gamma$.

\section{B. Mutual Information}

The focus of this paper is on the statistics of the mutual information of OFDM-based spatial multiplexing systems. It is now well-known that the instantaneous mutual information in $\mathrm{b} / \mathrm{s} / \mathrm{Hz}$ for a given channel realization is given by [10]

$$
\mathcal{I}_{\text {ofdm }}=\frac{1}{N} \sum_{k=0}^{N-1} \mathcal{I}_{k}
$$

where $\mathcal{I}_{k}$ is the instantaneous mutual information for the $k$ th OFDM subcarrier, given by

$$
\mathcal{I}_{k}=\log _{2} \operatorname{det}\left(\mathbf{I}_{N_{r}}+\frac{\gamma}{N_{t}} \mathbf{H}_{k} \mathbf{H}_{k}^{\dagger}\right) \text {. }
$$

Note that the loss in mutual information due to the cyclic prefix has been neglected in (6). The mean (ergodic) mutual information is given by

$$
E\left[\mathcal{I}_{\text {ofdm }}\right]=\frac{1}{N} \sum_{k=0}^{N-1} E\left[\mathcal{I}_{k}\right]
$$

which was shown in [10] to be the same as the ergodic mutual information of a flat-fading channel, in which case closedform expressions are now available [8,21].

\section{VARIANCE OF the Mutual Information}

In this section we derive new closed-form expressions for the variance of the mutual information of OFDM-based spatial multiplexing. Our results are exact, and apply for arbitrary finite system and channel parameters. We also present simplified expressions for the variance in the low SNR regime, and give explicit reduced variance expressions for the case of SISO systems. These results will be subsequently used in Section IV for providing accurate approximations to the distribution of the mutual information.

\section{A. Exact Analysis at All SNRs}

The following theorem presents an exact expression for the variance of the mutual information of MIMO-OFDM systems.

Theorem 1: The variance of the mutual information of MIMO-OFDM systems is given by

$$
\begin{array}{r}
\operatorname{Var}\left(\mathcal{I}_{\text {ofdm }}\right)=\frac{\left(\log _{2}(e)\right)^{2}}{\Gamma_{m}(n) \Gamma_{m}(m)}\left(\frac{2}{N^{2}} \sum_{d=1}^{N-1}(N-d) \varphi\left(\rho_{d}\right)\right. \\
\left.+\sum_{r=1}^{m} \sum_{s=1}^{m} \frac{\operatorname{det}\left(\mathbf{B}_{r, s}\right)}{N}-\frac{\left(\sum_{r=1}^{m} \operatorname{det}\left(\mathbf{A}_{r}\right)\right)^{2}}{\Gamma_{m}(n) \Gamma_{m}(m)}\right)
\end{array}
$$


where $m=\min \left(N_{r}, N_{t}\right), n=\max \left(N_{r}, N_{t}\right), \Gamma_{m}(n)=$ $\prod_{i=1}^{m} \Gamma(n-i+1)$ is the normalized complex multivariate gamma function, and

$\varphi\left(\rho_{d}\right)= \begin{cases}\frac{\left(\sum_{r=1}^{m} \operatorname{det}\left(\mathbf{A}_{r}\right)\right)^{2}}{\Gamma_{m}(n) \Gamma_{m}(m)}, & \left|\rho_{d}\right|=0 \\ \sum_{r=1}^{m} \sum_{s=1}^{m} e^{\frac{2 N_{t}}{\gamma}} \operatorname{det}\left(\mathbf{C}_{r, s}\left(\rho_{d}\right)\right), & 0<\left|\rho_{d}\right|<1 \\ \sum_{r=1}^{m} \sum_{s=1}^{m} \operatorname{det}\left(\mathbf{B}_{r, s}\right), & \left|\rho_{d}\right|=1\end{cases}$

$\mathbf{A}_{r}, \mathbf{B}_{r, s}$ and $\mathbf{C}_{r, s}(\cdot)$ are $m \times m$, with $(i, j)^{\text {th }}$ elements

$\left(\mathbf{A}_{r}\right)_{i, j}=\left\{\begin{array}{ll}b !, & j \neq r \\ b ! e^{N_{t} / \gamma} g_{1}(b+1), & j=r\end{array}\right.$,
$\left(\mathbf{B}_{r, s}\right)_{i, j}=\left\{\begin{array}{l}b !, \\ b ! e^{N_{t} / \gamma} g_{1}(b+1), \quad j \neq r \text { and } j \neq s \\ 2\left(N_{t} / \gamma\right)^{b+1} e^{N_{t} / \gamma} \sum_{t=0}^{b}\left(\begin{array}{l}b \\ t\end{array}\right)(-1)^{t} j=s, \text { and } r \neq s \\ \times \mathrm{G}_{3,4}^{4,0}\left(N_{t} /\left.\gamma\right|_{0, t-b-1, t-b-1, t-b-1} ^{t-b, t-b,-b}\right), \quad j=r=s\end{array}\right.$

and

$\left(\mathbf{C}_{r, s}\left(\rho_{d}\right)\right)_{i, j}= \begin{cases}\eta_{i, j}\left(1, \rho_{d}\right) & i \neq r, j \neq s \\ \eta_{i, j}\left(g_{1}(z), \rho_{d}\right) & i=r, j \neq s \\ \left|\rho_{d}\right|^{2(i-j)} \eta_{j, i}\left(g_{1}(z), \rho_{d}\right) & i \neq r, j=s \\ \frac{\left(1-\left|\rho_{d}\right|^{2}\right)^{z}}{\mid \frac{2 N_{t}\left|\rho_{d}\right|^{2}}{\gamma\left(1-\left|\rho_{d}\right|^{2}\right)}} & \\ \times \sum_{t=0}^{\infty} \frac{\left|\rho_{d}\right|^{2 t} \Gamma(u) \Gamma(v) g_{2}(u) g_{2}(v)}{t !(\tau+t) !} & i=r, j=s\end{cases}$

respectively. Also, $b=n+m-i-j, \tau=n-m, z=$ $\tau+i+j-1, u=\tau+i+t, v=\tau+j+t$, and $\mathrm{G}_{3,4}^{4,0}(\cdot)$ is the Meijer-G function [22, eq. (9.301)],

$$
\begin{aligned}
& g_{1}(z)=\sum_{h=1}^{z} \mathrm{E}_{h}\left(\frac{N_{t}}{\gamma}\right) \\
& g_{2}(z)=\sum_{h=1}^{z} \mathrm{E}_{h}\left(\frac{N_{t}}{\gamma\left(1-\left|\rho_{d}\right|^{2}\right)}\right)
\end{aligned}
$$

where $\mathrm{E}_{h}(\cdot)$ is the Exponential Integral [23, eq. (5.1.12)]. The function $\eta_{i, j}(\cdot, \cdot)$ is defined as

$$
\begin{aligned}
\eta_{i, j}\left(f(z), \rho_{d}\right)=\Gamma(\tau & +j) \sum_{t=0}^{j-1}\left(\begin{array}{c}
j-1 \\
t
\end{array}\right)\left(\frac{1-\left|\rho_{d}\right|^{2}}{\left|\rho_{d}\right|^{2}}\right)^{t} \\
& \times(\tau+j-t)_{i-1} f(z-t)
\end{aligned}
$$

for an arbitrary input function $f$, and $(a)_{r}=a(a+1) \cdots(a+$ $r-1),(a)_{0}=1$, is the Pochammer symbol.

Proof: See the Appendix.

We note that although the exact variance expression in Theorem 1 involves infinite series of exponential integrals, its numerical evaluation can be made more efficient by exploiting the recurrence relations [23, eqs. (5.1.7) and (5.1.14)], and as such, only a single exponential integral must be explicitly evaluated when summing these series.

The following corollary presents a simple exact variance expression for the mutual information of SISO OFDM systems (i.e. $m=1, n=1$ ). To the best of our knowledge, this result is also new.

Corollary 1: The variance of the mutual information of SISO-OFDM systems is given by

$$
\operatorname{Var}\left(\mathcal{I}_{\text {ofdm }}\right)=\left(\log _{2}(e)\right)^{2}\left(\frac{2}{N^{2}} \sum_{d=1}^{N-1}(N-d) \varphi\left(\rho_{d}\right)\right.
$$

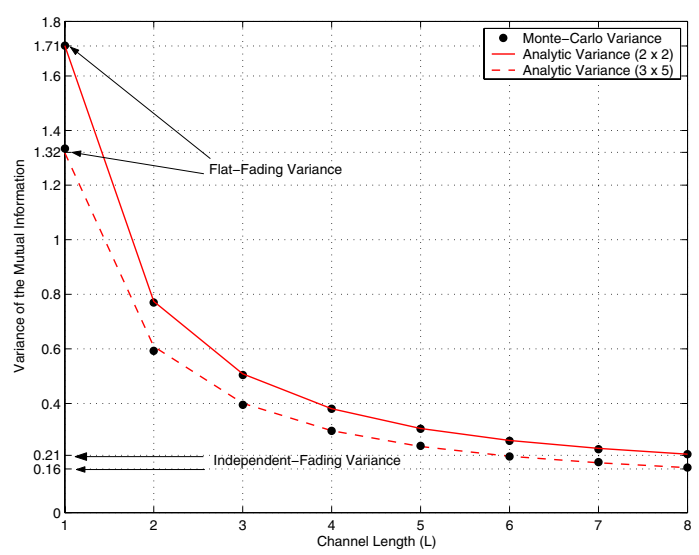

Fig. 2. Variance of the mutual information of MIMO-OFDM for different $N_{t} \times N_{r}$ antenna configurations, and different channel lengths (uniform power delay profile). The "Analytic Variance" curves are based on (8). 8 subcarriers is considered, with SNR of $10 \mathrm{~dB}$.

$$
\left.+\frac{2 e^{1 / \gamma}}{\gamma N} \mathrm{G}_{3,4}^{4,0}\left(1 /\left.\gamma\right|_{0,-1,-1,-1} ^{0,0,0}\right)-e^{2 / \gamma}\left(g_{1}(1)\right)^{2}\right)
$$

where

$$
\varphi\left(\rho_{d}\right)= \begin{cases}e^{2 / \gamma}\left(g_{1}(1)\right)^{2}, & \left|\rho_{d}\right|=0 \\ \left(1-\left|\rho_{d}\right|^{2}\right) e^{\frac{2}{\gamma\left(1-\left|\rho_{d}\right|^{2}\right)}} & \\ \quad \times \sum_{t=0}^{\infty}\left|\rho_{d}\right|^{2 t}\left(g_{2}(1+t)\right)^{2}, & 0<\left|\rho_{d}\right|<1 \\ \frac{2 e^{1 / \gamma}}{\gamma N} \mathrm{G}_{3,4}^{4,0}\left(1 /\left.\gamma\right|_{0,-1,-1,-1} ^{0,0,0}\right), & \left|\rho_{d}\right|=1\end{cases}
$$

In Fig. 2 we compare the analytical variance expression (8) with the variance obtained via Monte-Carlo simulation. Results are presented for two different $N_{t} \times N_{r}$ antenna configurations as a function of the channel length $L$. A uniform power delay profile is assumed (i.e. $\sigma_{p}^{2}=1 / L$, for $p=0, \ldots L-1$ ), 8 subcarriers are used (simply by way of example; similar results are obtained for higher numbers of subcarriers), and the SNR is set to $10 \mathrm{~dB}$. In all cases we see a precise agreement between the simulated and analytic curves. Moreover, the variance is seen to be largest for the system with the least antennas, regardless of the channel length. For both antenna configurations, we see that the variance reduces with increasing $L$, and that this reduction is most significant for small $L$. For example, by increasing the channel length from $L=1$ (flat-fading) to $L=2$, the variance for both antenna configurations is more than halved.

\section{B. Analysis at Low SNR}

The following theorem presents a very simple closed-form expression for the variance of the mutual information of MIMO-OFDM in the low SNR regime.

Theorem 2: In the low SNR regime, the variance of the mutual information of MIMO-OFDM systems is given by

$\operatorname{Var}^{0}\left(\mathcal{I}_{\text {ofdm }}\right)=\left(\log _{2}(e)\right)^{2} \frac{\gamma^{2} N_{r}}{N N_{t}}\left(1+2 \sum_{d=1}^{N-1} \frac{N-d}{N}\left|\rho_{d}\right|^{2}\right)$.

Proof: Omitted due to space constraints. A complete derivation is given in the extended journal version [24]. 


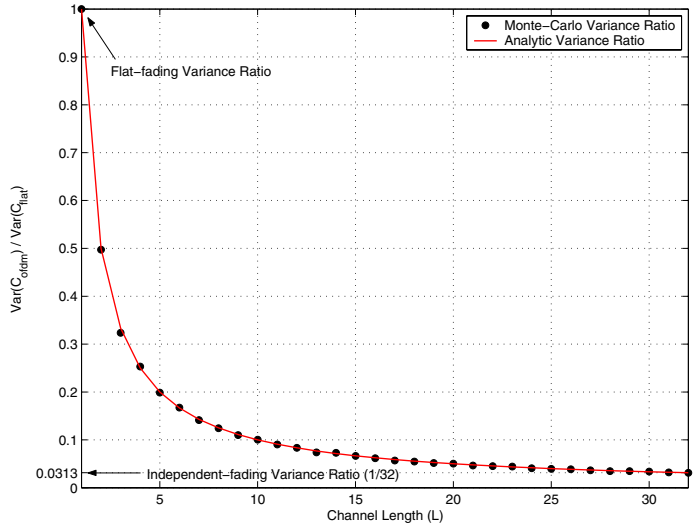

Fig. 3. Ratio of the MIMO-OFDM mutual information variance and the flatfading MIMO mutual information variance at low SNR, for different channel lengths (uniform power delay profile). The "Analytic Variance Ratio" curve is based on (11). A $2 \times 2$ system at $-25 \mathrm{~dB}$ is considered with 32 subcarriers.

The following corollary gives upper and lower bounds for the variance of the MIMO-OFDM mutual information in the low SNR regime.

Corollary 2: In the low SNR regime, the variance of the mutual information of MIMO-OFDM systems satisfies

$$
\frac{1}{N} \leq \frac{\operatorname{Var}^{0}\left(\mathcal{I}_{\text {ofdm }}\right)}{\operatorname{Var}^{0}\left(\mathcal{I}_{\text {flat }}\right)}=\frac{1}{N}\left(1+2 \sum_{d=1}^{N-1} \frac{N-d}{N}\left|\rho_{d}\right|^{2}\right) \leq 1
$$

where $\operatorname{Var}^{0}\left(\mathcal{I}_{\text {flat }}\right)$ denotes the mutual information variance for an i.i.d. flat-fading Rayleigh MIMO channel. The left-hand side is an equality for $\left|\rho_{d}\right|=0$ (independent fading across all subcarriers), and the right-hand side is an equality for $\left|\rho_{d}\right|=1$ (identical fading across all subcarriers, i.e. flat-fading).

Proof: The proof follows by using $0 \leq\left|\rho_{d}\right| \leq 1$ in (10), and noting that $\operatorname{Var}^{0}\left(\mathcal{I}_{\text {flat }}\right)=\left(\log _{2}(e)\right)^{2} \frac{\gamma^{2} N_{r}}{N_{t}}$, which is found by directly setting $N=1$ in (10).

It is interesting to note from (11) that in the low SNR regime, the scaling of the MIMO-OFDM variance with respect to the flat-fading variance depends only on the channel delay profile, and is independent of the number of antennas.

\section{APPROXIMATIONS FOR THE CAPACITY Distribution}

We now use the analytic expressions from the previous section to present and investigate approximations for the distribution of mutual information. We model the channel according to the exponential power delay profile [25]

$$
\sigma_{p}^{2}= \begin{cases}\frac{1-e^{-1 / K_{\exp }}}{1-e^{-L / K_{\exp }}} e^{-p / K_{\exp }} & \text { for } 0 \leq p<L \\ 0 & \text { otherwise }\end{cases}
$$

where $K_{\exp }$ characterizes the rate of decay of the power delay profile as a function of $p$, and is loosely related to the r.m.s. delay spread [25].

We first investigate a Gaussian approximation.

Fig. 4 presents the analytical Gaussian approximation for the MIMO-OFDM mutual information p.d.f. based on the exact mean and variance expressions in (17) and (1) respectively, as well as empirically generated p.d.f.s (Monte-

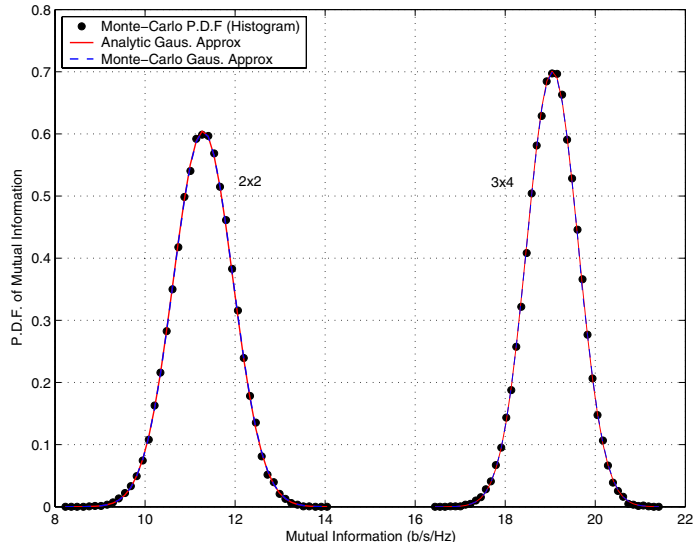

Fig. 4. P.d.f. of MIMO-OFDM mutual information for different $N_{t} \times N_{r}$ antenna configurations. The "Analytic Gaus. Approx." curves are based on the exact mean formula (17) and exact variance formula (8). 64 subcarriers is considered, with SNR of $20 \mathrm{~dB}$. The channel follows an 8-path exponential power delay profile with $K_{\exp }=4$.

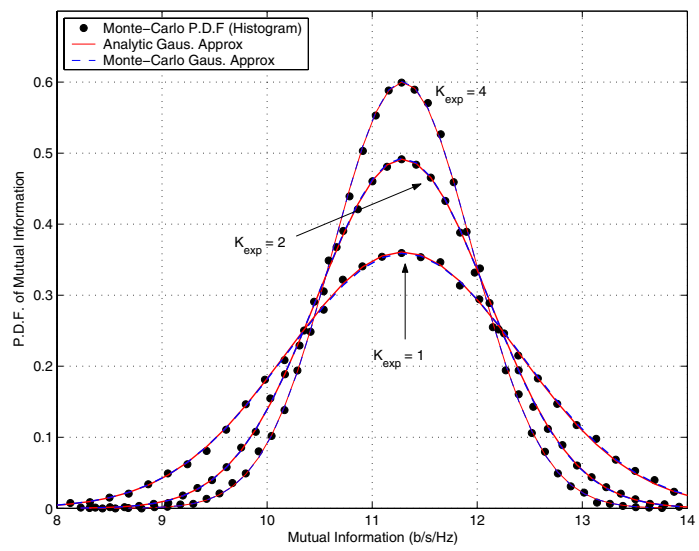

Fig. 5. P.d.f. of MIMO-OFDM mutual information for different rms delay spreads (channels follow an 8-path exponential power delay profile, with different $\left.K_{\text {exp }}\right)$. The "Analytic Gaus. Approx." curves are based on the exact mean formula (17) and exact variance formula (8). A $2 \times 2$ system with 16 subcarriers is considered, with SNR of $20 \mathrm{~dB}$.

Carlo histogram), for different antenna configurations. A 64subcarrier system is considered with SNR of $20 \mathrm{~dB}$. We see that the analytic curves match the true distribution almost perfectly for both antenna configurations. We also present curves for a simulation based Gaussian approximation (based on the mean and variance of the Monte-Carlo generated histograms) for further verification. Note that these curves are indistinguishable from our new analytical Gaussian approximation curves.

Fig. 5 compares the analytical Gaussian approximation with empirically-generated p.d.f. curves, for different channel rms delay spreads. Again we see that the analytic Gaussian approximation is accurate in all cases. Moreover, we see a significant reduction in the variance of the mutual information as the rms delay spread increases (i.e. as $K_{\exp }$ increases). Again note that the Monte-Carlo Gaussian approximation is indistinguishable from our new analytical curves.

Fig. 6 presents the distribution of the mutual information at low SNRs. The analytic Gaussian approximation curve is 


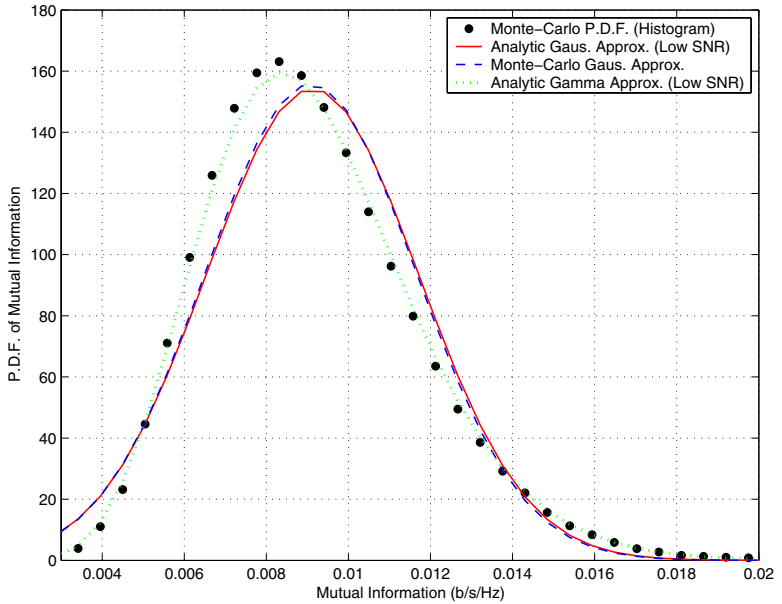

Fig. 6. P.d.f. of MIMO-OFDM mutual information at low SNR. The "Analytic Gaus. Approx (Low SNR)" and "Analytic Gamma Approx (Low SNR)" curves are based on the low SNR mean formula obtained by combining [24, Eq. 131] and [24, Eq. 133], and the low SNR variance formula (10). A $2 \times 2$ system with 32 subcarriers is considered, with SNR of $-25 \mathrm{~dB}$. The channel follows a 4 -path exponential power delay profile with $K_{\exp }=2$.

generated based on the low SNR mean formula obtained by combining [24, Eq. 131] and [24, Eq. 133], and the low SNR variance formula (10). In this case we see that a Gaussian distribution no longer accurately predicts the mutual information p.d.f. This can be explained by examining the Taylor expansion of (6) around $\gamma=0$ :

$$
\mathcal{I}_{k}=\log _{2}(e) \frac{\gamma}{N_{t}} \operatorname{tr}\left(\mathbf{H}_{k} \mathbf{H}_{k}^{\dagger}\right)+o(\gamma) .
$$

We see that at low SNRs, the mutual information for each subcarrier is a function of $\operatorname{tr}\left(\mathbf{H}_{k} \mathbf{H}_{k}^{\dagger}\right)$, which for i.i.d. Rayleigh fading is $\sim \chi_{2 N_{r} N_{t}}^{2}$. Hence, the overall mutual information (7) is distributed as the sum of $N$ correlated $\chi_{2 N_{r} N_{t}}^{2}$ random variables which (for small $N$ ), is clearly different to Gaussian.

Motivated by this observation, we propose to approximate the mutual information p.d.f. at low SNR with a Gamma distribution. Note that a Gamma approximation was previously considered in the context of flat-fading channels in [26]. The Gamma p.d.f. is given by

$$
f(x)=\frac{\theta^{r} x^{r-1} e^{-\theta x}}{\Gamma(r)}, \quad x \geq 0
$$

where $r$ is the shape parameters and $\theta$ is the scale parameter. By matching the first two moments, a Gamma approximation for the mutual information p.d.f. of MIMO-OFDM is obtained by evaluating $r=\frac{E\left[\mathcal{I}_{\text {ofdm }}\right]}{\operatorname{Var}\left(\mathcal{I}_{\text {ofd } m}\right)}$ and $\theta=\frac{E^{2}\left[\mathcal{I}_{\text {ofdm }}\right]}{\operatorname{Var}\left(\mathcal{I}_{\text {ofdm }}\right)}$. This analytic Gamma approximation is plotted in Fig. 6, based on the same low SNR analytic mean and variance formulas as used for the low SNR Gaussian approximation above. We clearly see that the Gamma approximation is much more accurate than the Gaussian approximation in this low SNR regime, and follows the simulated p.d.f. very closely.

\section{Conclusions}

We have presented new closed-form approximations to the capacity distribution of frequency-selective MIMO channels, in the context of OFDM-based spatial multiplexing systems. These approximations were based on new exact closed-form expressions which were derived for the variance of the mutual information, applying for arbitrary finite system and channel parameters. We showed that for many scenarios a Gaussian approximation is accurate, whilst at low SNR a Gamma approximation yielded even higher accuracy.

\section{APPENDIX}

Proof: By the definition of variance, we have

$$
\operatorname{Var}\left(\mathcal{I}_{\text {ofdm }}\right)=E\left[\mathcal{I}_{\text {ofdm }}^{2}\right]-E^{2}\left[\mathcal{I}_{\text {ofdm }}\right] .
$$

Note that, under the assumptions in Section II-A, the channel statistics for each subcarrier (and therefore, the mutual information statistics) are identical [10], and moreover, these statistics are equal to that of a flat-fading i.i.d. Rayleigh channel. Thus, and using (5), (15) can be written as

$$
\begin{aligned}
\operatorname{Var}\left(\mathcal{I}_{\text {ofdm }}\right)=\frac{1}{N^{2}} & \left(\sum_{k=0}^{N-1} \sum_{\ell=0, \ell \neq k}^{N-1} E\left[\mathcal{I}_{k} \mathcal{I}_{\ell}\right]\right) \\
& +\frac{1}{N} E\left[\mathcal{I}_{\text {flat }}^{2}\right]-E^{2}\left[\mathcal{I}_{\text {flat }}\right]
\end{aligned}
$$

where $\mathcal{I}_{\text {flat }}$ is the mutual information of a flat-fading channel.

The first and second moments of the mutual information for flat-fading channels has been previously derived in [8, eqs. (29) and (31)]. Using [23, eq. (6.5.9)], we perform some basic manipulations to express these results in alternative simplified forms as follows

$$
\begin{aligned}
& E\left[\mathcal{I}_{\text {flat }}\right]=\frac{\log _{2}(e)}{\Gamma_{m}(n) \Gamma_{m}(m)} \sum_{r=1}^{m} \operatorname{det}\left(\mathbf{A}_{r}\right) \\
& E\left[\mathcal{I}_{\text {flat }}^{2}\right]=\frac{\left(\log _{2}(e)\right)^{2}}{\Gamma_{m}(n) \Gamma_{m}(m)} \sum_{r=1}^{m} \sum_{s=1}^{m} \operatorname{det}\left(\mathbf{B}_{r, s}\right)
\end{aligned}
$$

where $\mathbf{A}_{r}$ and $\mathbf{B}_{r, s}$ are defined in the theorem.

The challenge is to evaluate the cross-correlation of the mutual information across frequency subcarriers $E\left[\mathcal{I}_{k} \mathcal{I}_{\ell}\right]$ which, using (6), can be written

$$
E\left[\mathcal{I}_{k} \mathcal{I}_{\ell}\right]=E\left[\sum_{i=1}^{m} \sum_{j=1}^{m} \alpha\left(\lambda_{i}\right) \alpha\left(\omega_{j}\right)\right]
$$

where $\underline{\lambda}=\left\{\lambda_{i}\right\}_{i=1}^{m}$ and $\underline{\omega}=\left\{\omega_{i}\right\}_{i=1}^{m}$ are the non-zero eigenvalues of $\mathbf{H}_{k} \mathbf{H}_{k}^{\dagger}$ and $\overline{\mathbf{H}}_{\ell} \mathbf{H}_{\ell}^{\dagger}$ respectively, and

$$
\alpha\left(\lambda_{i}\right)=\log _{2}\left(1+\frac{\gamma}{N_{t}} \lambda_{i}\right) .
$$

Now let $\lambda$ and $\omega$ be randomly (uniformly) chosen eigenvalues from $\underline{\lambda}$ and $\underline{\omega}$ respectively. Then

$$
\operatorname{Pr}\left(\alpha(\lambda) \alpha(\omega)=\alpha\left(\lambda_{i}\right) \alpha\left(\omega_{j}\right)\right)=\frac{1}{m^{2}}, \quad \forall i, j=1, \ldots, m,
$$

and hence

$$
E[\alpha(\lambda) \alpha(\omega)]=\frac{1}{m^{2}} \sum_{i=1}^{m} \sum_{j=1}^{m} E\left[\alpha\left(\lambda_{i}\right) \alpha\left(\lambda_{j}\right)\right],
$$




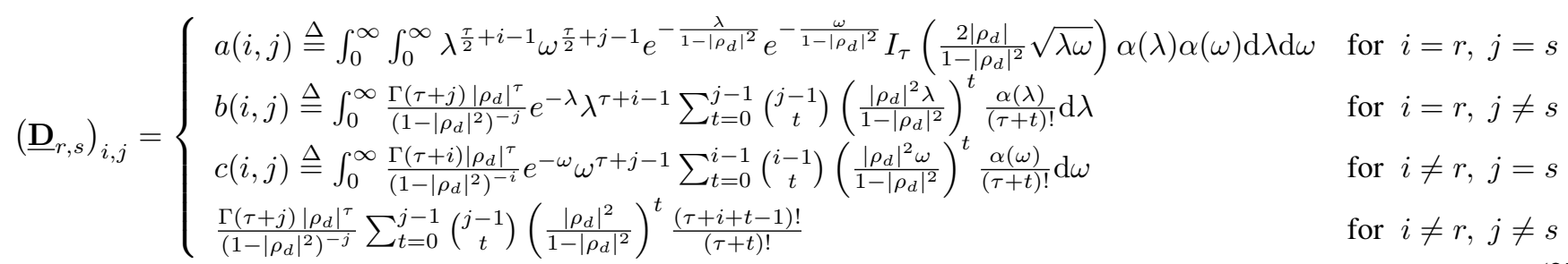

so it follows that (19) can be written as

$$
E\left[\mathcal{I}_{k} \mathcal{I}_{\ell}\right]=m^{2} E[\alpha(\lambda) \alpha(\omega)] .
$$

Now we substitute (23) into (16) and, after extensive manipulations (see journal version [24]), evaluate the left-hand summation in (16) as follows

$$
\sum_{k=0}^{N-1} \sum_{\ell=0, \ell \neq k}^{N-1} E\left[\mathcal{I}_{k} \mathcal{I}_{\ell}\right]=2 \sum_{d=1}^{N-1}(N-d) E\left[\mathcal{I}_{0} \mathcal{I}_{d}\right]
$$

where, for the cases $\rho_{d}=0$ and $\rho_{d}=1$,

$$
\begin{array}{lll}
E\left[\mathcal{I}_{0} \mathcal{I}_{d}\right]=E^{2}\left[\mathcal{I}_{\text {flat }}\right], & & \rho_{d}=0 \\
E\left[\mathcal{I}_{0} \mathcal{I}_{d}\right]=E\left[\mathcal{I}_{\text {flat }}^{2}\right], & & \rho_{d}=1
\end{array}
$$

and, for the case $0<\left|\rho_{d}\right|<1$,

$$
E\left[\mathcal{I}_{0} \mathcal{I}_{d}\right]=\frac{\left|\rho_{d}\right|^{-m(n-1)}}{\Gamma_{m}(n) \Gamma_{m}(m)\left(1-\left|\rho_{d}\right|^{2}\right)^{m}} \sum_{r=1}^{m} \sum_{s=1}^{m} \operatorname{det}\left(\underline{\mathbf{D}}_{r, s}\right)
$$

where $\underline{\mathbf{D}}_{r, s}$ is an $m \times m$ matrix with $(i, j)^{\text {th }}$ elements given in (27). We now evaluate $b(i, j)$ and $c(i, j)$ in closed-form using the identity [4]

$$
\int_{0}^{\infty} \frac{\ln (1+\alpha \lambda) \lambda^{q-1}}{e^{b \lambda}} \mathrm{d} \lambda=\frac{\Gamma(q) e^{b / \alpha}}{b^{q}} \sum_{h=1}^{q} \mathrm{E}_{h}\left(\frac{b}{\alpha}\right) .
$$

We evaluate the remaining integral, $a(i, j)$, by expressing $I_{\tau}(\cdot)$ as a power series, and then integrating term by term using (28). The result then follows by substituting (24), (18), and (17) into (16) and simplifying.

\section{REFERENCES}

[1] İ. E. Telatar, "Capacity of multi-antenna Gaussian channels," Europ. Trans. Commun., pp. 585-595, Nov-Dec. 1999.

[2] G. J. Foschini, "Layered space-time architecture for wireless communications in a fading environment when using multi-element antennas," Bell Labs Tech. J., vol. 1, no. 2, pp. 41-59, Autumn 1996.

[3] P. J. Smith, S. Roy, and M. Shafi, "Capacity of MIMO systems with semicorrelated flat fading," IEEE Trans. Inform. Theory, vol. 49, no. 10, pp. 2781-2788, Oct 2003.

[4] G. Alfano, A. Lozano, A. M. Tulino, and S. Verdú, "Mutual information and eigenvalue distribution of MIMO Ricean channels," in Int. Symp. on Info. Theory and Appl. (ISITA), Parma, Italy, Oct 10-13 2004.

[5] M. Kiessling and J. Speidel, "Mutual information of MIMO channels in correlated Rayleigh fading environments - a general solution," in IEEE Int. Conf. on Commun. (ICC), Paris, France, Jun 2004, pp. 814-818.

[6] M. R. McKay and I. B. Collings, "General capacity bounds for spatially correlated Rician MIMO channels," IEEE Trans. Inform. Theory, vol. 51, no. 9, pp. 3121-3145, Sept. 2005.

[7] A. M. Tulino, A. Lozano, and S. Verdú, "Impact of antenna correlation on the capacity of multiantenna channels," IEEE Trans. Inform. Theory, vol. 51, no. 7, pp. 2491-2509, Jul 2005.
[8] M. Kang and M.-S. Alouini, "Capacity of MIMO Rician channels," IEEE Trans. Wireless Commun., vol. 5, no. 1, pp. 112-122, Jan 2006.

[9] M. R. McKay and I. B. Collings, "Improved general lower bound for spatially-correlated Rician MIMO capacity," IEEE Commun. Lett., pp. 162-164, Mar 2006.

[10] H. Bölcskei, D. Gesbert, and A. J. Paulraj, "On the capacity of OFDMbased spatial multiplexing systems," IEEE Trans. Commun., vol. 50, no. 2, pp. 225-234, Feb. 2002

[11] P. J. Smith, L. M. Garth, and S. Loyka, "Exact capacity distributions for MIMO systems with small numbers of antennas," IEEE Commun. Lett., vol. 7, no. 10, pp. 481-483, Oct 2003.

[12] P. J. Smith and L. M. Garth, "Exact capacity distribution for dual MIMO systems in Ricean fading," IEEE Commun. Lett., vol. 8, no. 1, pp. 1820, Jan 2004

[13] O. Oyman, R. U. Nabar, H. Bölcskei, and A. J. Paulraj, "Characterizing the statistical properties of mutual information in MIMO channels," IEEE Trans. Signal Proc., vol. 51, no. 11, pp. 2784-2795, Nov 2003.

[14] K. Liu, V. Raghavan, and A. M. Sayeed, "Capacity scaling and spectral efficiency in wide-band correlated MIMO channels," IEEE Trans. Inform. Theory, vol. 49, pp. 2504-2526, Oct 2003.

[15] L. S. Pillutla and S. K. Jayaweera, "MIMO capacity of an OFDM-based system under Ricean fading," in Proc. IEEE Veh. Tech. Conf. (VTC '04), Milan, Italy, May 2004, pp. 618-622

[16] M. R. McKay and I. B. Collings, "On the capacity of frequencyflat and frequency-selective Rician MIMO channels with single-ended correlation," IEEE Trans. Wireless Commun., vol. 5, no. 8, pp. 20382043, Aug 2006.

[17] H. A. Suraweera, J. T. Y. Ho, T. Sivahumaran, and J. Armstrong, "An approximated Gaussian analysis and results on the capacity distribution for MIMO-OFDM," in Proc. IEEE Int. Symp. Personal, Indoor and Mobile Radio Commun. (PIMRC '05), Berlin, Germany, Sept 2005.

[18] G. Barriac and U. Madhow, "Characterizing outage rates for spacetime communication over wideband channels," IEEE Trans. Commun. vol. 52 , no. 12 , pp. $2198-2207$, Dec. 2004.

[19] A. L. Moustakas and S. H. Simon, "On the outage capacity of correlated multiple-path MIMO channels," IEEE Trans. Inform. Theory, 2006, submitted for publication, available at http://arxiv.org/abs/cs.IT/0509093.

[20] Y. Li, L. J. Cimini, and N. R. Sollenberger, "Robust channel estimation for OFDM systems with rapid dispersive fading channels," IEEE Trans. Commun., vol. 46, no. 7, pp. 902-915, July 1998.

[21] H. Shin and J. H. Lee, "Capacity of multiple-antenna fading channels: Spatial fading correlation, double scattering, and keyhole," IEEE Trans. Inform. Theory, vol. 49, no. 10, pp. 2636-2647, Oct 2003.

[22] I. S. Gradshteyn and I. M. Ryzhik, Table of Integrals, Series, and Products, 4th ed. San Diego, CA: Academic, 1965.

[23] M. Abramowitz and I. A. Stegun, Handbook of Mathematical Functions with Formulas, Graphs, and Mathematical Tables, 4th ed. New York: Dover Publications, 1965.

[24] M. R. McKay, P. J. Smith, H. A. Suraweera, and I. B. Collings, "On the capacity distribution of OFDM-based spatial multiplexing: Exact variance and outage approximation," IEEE Trans. Inform. Theory, 2006 , submitted.

[25] S. H. Müller-Weinfurtner, "Coding approaches for multiple antenna transmission in fast fading and OFDM," IEEE Trans. Signal Processing, vol. 50, no. 10, pp. 2442-2450, Oct. 2002.

[26] M. Kang, L. Yang, and M.-S. Alouini, "How accurate are the Gaussian and Gamma approximations to the outage capacity of MIMO channels," in Proc. of Sixth Baiona Workshop on Sig. Proc. in Commun., Baiona, Spain, Sept. 2003. 